\title{
Prevalence and Determinants of Home Delivery in Urban and Rural Philippines: Evidence From the 2017 National Demographic and Health Survey
}

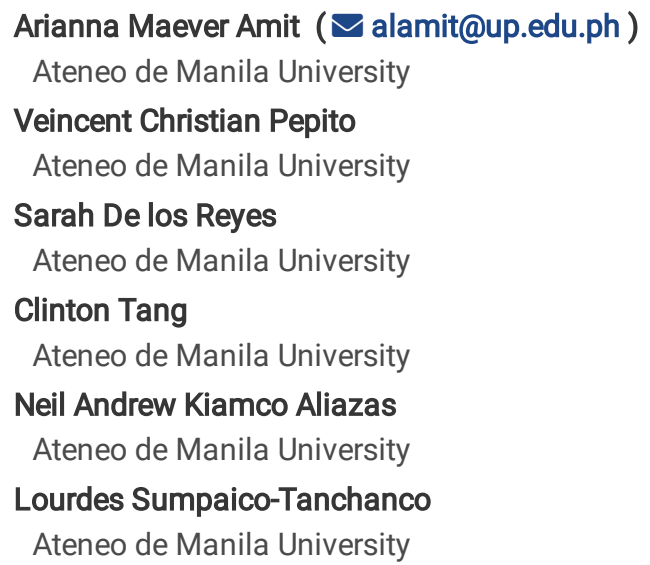

\section{Research Article}

Keywords: childbirth, demographic health survey, home delivery, institutional delivery, sustainable development goals, Philippines

Posted Date: June 16th, 2021

DOI: https://doi.org/10.21203/rs.3.rs-599893/v1

License: (c) This work is licensed under a Creative Commons Attribution 4.0 International License. Read Full License 


\section{Abstract}

Background: Women's choice of place of delivery has implications on rates of maternal and child mortality. Despite notable efforts to improve maternal health in the Philippines, disparities in the utilization of health services during pregnancy and delivery persist between urban and rural communities. This study aims to provide an updated and detailed comparison of prevalence and determinants of home delivery in the Philippines, and in urban and rural communities in the country.

Methods: We used data from the 2017 Philippine National Demographic and Health Survey (NDHS). A total of 3,084 married or cohabiting women 15 to 49 years old with one birth in the preceding year of the survey were included in the analyses. Logistic regression methods for survey data were used to identify determinants for the choice of home-based childbirth over institutional delivery in the Philippines, and in urban and rural communities.

Results: There remains a considerable proportion of 3,084 married or cohabiting women aged 15 to 49 years old with one birth in the preceding year of the survey who delivered at home [18.97\% (95\% Cl: 16.04, 22.28)]. More women in rural areas delivered at home [22.95\% $(95 \% \mathrm{Cl}: 18.93,27.54)]$ than their counterparts in urban areas [13.49\% (95\% Cl: 9.59, 18.66)], reflecting a significant difference in the home delivery prevalence of women relative to their place of residence. Our regression analyses for all three populations consistently showed that socio-economic, individual, and pregnancy-related factors influence a mother's decision to deliver at home. Wealth index has the most pronounced effect with a significant increase in odds of home delivery among urban and rural women of the lowest wealth categories.

Conclusions: There are marked improvements in reducing maternal and child mortality in the Philippines, reflecting progress in health care services. However, our findings suggest that current strategies need to be revisited to substantially reduce preventable deaths associated with childbirth, particularly in the context of birthing location, and narrow the gap between urban and rural women. Innovative interventions targeting women with specific characteristics as reported in our study are needed. Efforts should also be made to contextualize and co-create innovations and solutions that will motivate women to deliver in health facilities.

\section{Background}

The global health prioritization of improving maternal health outcomes and child survival has largely been successful [1, 2]. From 2000 to 2015, world leaders committed to the Millennium Development Goals (MDG) that aimed to meet development- and health-related targets [3]. This included MDG 4 that targeted a two-third reduction in child mortality and MDG 5 that aimed for a 75\% reduction in the maternal mortality ratio (MMR) [3, 4]. Many developing countries however failed to meet the ambitious MDG targets, and this has given way to the subsequent maternal mortality- and child mortality-related targets for the Sustainable Development Goals (SDG) $[1,2,5]$. The global MMR declined by $43.9 \%$ with 303,000 maternal deaths in 2015 , but progress needs to be accelerated to meet the SDG target of a $75 \%$ reduction in MMR between 2016 and 2030 [1, 6]. Similarly, global child mortality rates declined by 64\% from 17.6 million in 1970 to 6.3 million under-5 children deaths in 2013 [2]. However, only developed countries achieved MDG 4 with other countries far behind and seemingly far from meeting the SDG target of ending preventable deaths of newborns and children under 5 years of age $[2,6]$.

The intrapartum care strategy for women centers healthcare around women's needs to optimize experience of labor and childbirth [7, 8]. This approach recognizes that most maternal deaths occur during labor, delivery, or 24 hours postpartum, and complications are not predictable, thus requiring care at a health facility [8]. It further emphasizes the importance of institutional deliveries, but at the same time allows a positive birthing experience. There is general consensus that delivery at a health facility is effective in bringing down high rates of maternal and child mortality $[7,9]$, and this is supported by interventions and policies instituted locally by countries. The Philippines makes for an interesting case study as it continues to fall behind the SDG targets for both women and children despite substantial improvements in facility-based deliveries and coverage of skilled attendants $[1,2,5,10]$.

The Philippines is an archipelagic low- and middle-income country with its health system transitioning towards universal health care that promises major reforms in the health sector to provide equitable access to health care for all Filipinos [11]. Prior the passage of Republic Act 11223 or "An Act Instituting Universal Health Care (UHC)" in 2019, resources and health capacity were poorly and unequally distributed with most concentrated in urban areas as evidenced in key maternal and child health indicators [2, 5, 9-12]. For example, the Philippines is one of the countries that accounts for $80 \%$ of child deaths worldwide [2]. It is also one of the countries with a low relative reduction in MMR from 1990 to 2015 with the latest available data suggesting that there are 121 maternal deaths per 100,000 live births $[1,13]$. More importantly, evidence suggests that $96 \%$ women who belonged to higher income classes delivered their child with a skilled provider increasing chances of survival, but this was only $42 \%$ for women in the poorest quintile [14]. Recognizing the importance of maternal health and the need to address related issues, the Philippine Department of Health (DOH) launched the Women's Health and Safe Motherhood Project 2 in 2006, the Maternal, Newborn, Child Health and Nutrition (MNCHN) strategy in 2014 with focus on priority groups such as those in low-resource areas $[9,15]$. Because maternal, neonatal, and child mortalities can be prevented with timely receipt of appropriate emergency obstetric care with as much 
as $73 \%$ of averted maternal deaths, strategies and recommendations are focused on encouraging women to deliver in health facilities $[9,15$, 16]. The MNCHN strategy and the UHC law both identify service delivery networks to improve coordination across all health care levels and access to health facilities $[9,11]$. Despite these however, women continue to deliver at home because of financial and environmental or geographic barriers, influences from networks, individual preferences, and social and cultural factors [17]. These need to be studied at a local context to effectively inform interventions and programs to accelerate progress in improving maternal health. Previous studies have focused on health care utilization of women in the Philippines [18-24]. However, there is a need to conduct a disaggregated analysis of women in urban and rural communities given the differences in availability and access to services, thus also requiring targeted interventions for each of these groups. This is especially important with the ongoing COVID-19 pandemic overwhelming the health system and further discouraging women to seek health care and services in health facilities. Because women are often disproportionately affected during disasters, further strengthening the health facilities across the country and integrating in the pandemic response strategies for women to birth safely will be critical to advancing progress to reduce maternal mortality $[1,25]$. In this paper, we thus aim to determine the factors influencing women's choice of delivering at home in the Philippines, and in urban and rural communities. In doing so, we can contextualize and co-create innovations and solutions that will motivate women to deliver in health facilities.

\section{Methods}

\section{Data sources}

The Demographic and Health Surveys (DHS) program provides estimates on demographic and health indicators. Part of the program, the Philippine National Demographic and Health Survey (NDHS) is a nationally representative survey that provides population, health, and nutrition data in the country. We used the latest 2017 Philippine NDHS dataset in our analysis [26].

The survey utilized a two-stage stratified sampling design referencing the Master Sampling Frame designed by the Philippine Statistics Authority. The first stage involved the selection of 1,250 primary sampling units (PSUs) which were barangays (or villages), portions of large barangays, or two or more adjacent small barangays. The second stage involved systematic random sampling of 20 or 26 housing units. Eligible participants were women 15 to 49 years old who resided in the households. The women's questionnaire in particular collected detailed information on birth history and child mortality, knowledge and use of family planning methods, fertility preferences, pregnancy, delivery, postnatal care, infant feeding practices, vaccinations and childhood illnesses, and other health issues with reference to the index child. Survey weights were computed to ensure representative estimates of all indicators. Additional information on the survey methodology is provided in the 2017 Philippine NDHS published report [26].

\section{Study population}

We described all women surveyed in the 2017 Philippine NDHS. For our analytic sample, we extracted a subset of the 2017 Philippine NDHS dataset using the following criteria: women of reproductive age (15-49 years) who had one child born the year before the survey, and are married or cohabiting. The basis for the criterion on marital status is that partner's educational attainment and women's decision-making power are significant determinants in women's choice for home delivery [19, 21, 27-31]. In the dataset, only married women and those living with their partners had data on decision-making power. This did not result to a large loss of data since only 170 single, widowed, separated, or divorced women with one birth the year preceding the survey had data on the outcome. The final sample size for this study is 3,084 women aged 15 to 49 years with one birth preceding the year of the survey and who were married or cohabiting with their partners. The births recode (PHBR71FL) file was used and we limited our regression analyses to women with no missing data in any of our study variables. To ensure that all models were comparable during model-building, we excluded from the multivariable analyses observations with missing data in any of the remaining variables of interest. Thus, only 1,945 respondents were included in the final analysis for the overall population, 585 respondents for the urban population, and 1,360 for the rural population.

\section{Study variables}

In this study, our primary outcome variable is place of delivery, which we dichotomized into 0 if the woman delivered at a health facility and 1 if she delivered at home. We make comparisons based on place of location (urban or rural) and build three separate regression models for each, namely: Philippines, urban, and rural. We selected potential determinants of home delivery based on evidence from previous studies and reviews, including socio-economic, individual, and pregnancy-related factors [17, 19, 21-23, 27-36]. Socio-economic factors included respondent's and partner's highest educational attainment, employment status, and wealth index. The wealth index was derived from a principal components analysis conducted by ICF International and was calculated as a score of ownership of household assets, durable goods, and household facilities. This index was classified into five categories with each household assigned to one of the categories: poorest, poorer, middle, richer, and richest. We used the combined wealth index for overall descriptive statistics, then recategorized into three because data for the richer and richest categories are sparse resulting to poor, middle, and rich categories. We used the wealth index separately computed for urban and rural populations for our urban-rural analyses. Similar categorizations were made. Individual factors included 
maternal age, marital status, and women's decision-making power as measured by who makes decisions about health care. Pregnancy-related factors focused on parity, birth order, number of antenatal care (ANC) visits and emergency preparedness during pregnancy. The operational definition of study variables is presented in Table 1.

Table 1

List of study variables and their operational definition

\begin{tabular}{|c|c|c|}
\hline Variable & Description & Categories \\
\hline \multicolumn{3}{|l|}{ Geographical factors } \\
\hline $\begin{array}{l}\text { Place of delivery } \\
\text { (Outcome) }\end{array}$ & Where the mother gave birth for the index child & Health facility, Home \\
\hline Place of residence & Type of place of residence & Philippines (overall), Urban, Rural \\
\hline \multicolumn{3}{|c|}{ Socio-economic factors } \\
\hline $\begin{array}{l}\text { Mother's } \\
\text { education }\end{array}$ & $\begin{array}{l}\text { Highest level of educational attainment of the } \\
\text { mother/respondent }\end{array}$ & $\begin{array}{l}\text { Higher education, Secondary education, Primary } \\
\text { education or lower }\end{array}$ \\
\hline $\begin{array}{l}\text { Partner's } \\
\text { education }\end{array}$ & $\begin{array}{l}\text { Highest level of educational attainment of respondent's } \\
\text { husband/partner }\end{array}$ & $\begin{array}{l}\text { Higher education, Secondary education, Primary } \\
\text { education or lower }\end{array}$ \\
\hline $\begin{array}{l}\text { Mother's } \\
\text { employment } \\
\text { status }\end{array}$ & Current working status of the mother/respondent & Employed, Unemployed \\
\hline Wealth index & $\begin{array}{l}\text { Derived variable using ownership of household assets, } \\
\text { durable goods, and household facilities }\end{array}$ & Rich, Middle, Poor \\
\hline \multicolumn{3}{|l|}{ Individual factors } \\
\hline Age & Current age of respondent at time of interview & Age in years (as is) \\
\hline Marital status & Current marital status of respondent at time of interview & Married, Cohabiting \\
\hline $\begin{array}{l}\text { Women's decision- } \\
\text { making power }\end{array}$ & Person who usually decides on respondent's health care & $\begin{array}{l}\text { Respondent alone; Both respondent and } \\
\text { husband/partner; Husband/partner, or someone else }\end{array}$ \\
\hline \multicolumn{3}{|c|}{ Pregnancy-related factors } \\
\hline Parity & Number of children ever born & Para one, Para $2-4$, Para 5 or more \\
\hline Birth order & Birth order of index child & Birth order (as is) \\
\hline ANC visits & $\begin{array}{l}\text { Adequate number ( } 4 \text { or more) of ANC visits as per } \\
\text { protocol }\end{array}$ & Adequate, Inadequate \\
\hline $\begin{array}{l}\text { Emergency } \\
\text { preparedness }\end{array}$ & Prepared money in case of emergency during pregnancy & Prepared, Not prepared \\
\hline
\end{tabular}

\section{Statistical analysis}

We performed all analyses using complex survey data analysis considering the primary sampling unit, stratum, and sample weight adjusting for selection probabilities. All our analyses are weighted, except for analyses that do not have an option for weighting. Proportions, means, odds ratios, except for tests of normality and rank-sum tests, are weighted. However, we still showed the number of observations, which are unweighted. We described our study variables using percentages for categorical variables and measures of central tendency for continuous variables for the overall population and urban-rural residence. We then assessed the association between dependent variables and independent variables in the Philippines and urban-rural residence using Pearson's chi-square test for categorical variables and Wilcoxon ranksum test for continuous variables. In the interest of maintaining a manageable number of variables to achieve parsimony in the multivariable model, we categorized our study variables into distal and proximal factors based on how closer they are in the causal pathway of association. We identified mother's and partner's highest educational attainment, working status, maternal age, and marital status as distal factors. We considered place of location for urban-rural models; wealth index, parity, birth order, women's decision-making power, emergency preparations during pregnancy, and ANC visits as proximal factors. This categorization is consistent with the findings reported in previous studies. For each potential determinant, we ran bivariate logistic regression separately with the outcome variable before carrying out the multivariable analyses. Study variables with p-value of less than 0.20 were entered into the multivariable models to determine factors associated with home delivery in the Philippines and in urban-rural settings. Distal factors were then regressed on proximal factors, and both distal and proximal risk factors were regressed on home delivery in the three separate models. We included variables with p-values of 0.05 in our final model. Variables that had $p$-values greater than 0.05 , but were important in the decision for home delivery based on literature $[17,19,21-23,27-36]$ were also 
retained in our final model. Only participants with complete data were included in our logistic regression analyses. We used Stata 14.0 IC for our analyses and set the level of significance at 0.05 [37]. We present the results of multivariable logistic regression for women with adjusted odds ratio (AOR) and $95 \%$ confidence interval (Cl) for each of the three models (Philippines, urban, and rural).

\section{Results}

\section{Participant characteristics}

In the total sample of 47,244 women aged 15-49 years, majority were married with a significant proportion of women and their partner's having finished at least secondary education. More than half were employed and were classified as having lower wealth index scores. A greater proportion of women in rural communities were married and employed, and finished at least secondary education with a partner who similarly had a secondary-level of education. Fewer women in rural communities belong to the high-income classes [Table 2].

Table 2

Characteristics of women 15-49 years of age, 2017 Philippine NDHS ( $n=47,244)$

\begin{tabular}{|c|c|c|c|c|c|c|}
\hline \multirow[t]{2}{*}{ Characteristic } & \multicolumn{2}{|c|}{ Philippines $(n=47,244)$} & \multicolumn{2}{|c|}{ Urban $(n=14,240)$} & \multicolumn{2}{|c|}{ Rural $(n=33,004)$} \\
\hline & $\mathbf{N}^{\mathbf{a}}$ & $\%(95 \% \mathrm{Cl})$ & $\mathbf{N}^{\mathrm{a}}$ & $\%(95 \% \mathrm{Cl})$ & $\mathrm{N}^{\mathrm{a}}$ & $\%(95 \% \mathrm{Cl})$ \\
\hline Age (Median, IQR) & \multicolumn{2}{|c|}{$38(32-44)$} & \multicolumn{2}{|c|}{$38(31-44)$} & \multicolumn{2}{|c|}{$38(32-44)$} \\
\hline \multicolumn{7}{|l|}{ Marital status } \\
\hline Single & 994 & $2.09(1.81,2.42)$ & 432 & $3.05(2.51,3.70)$ & 562 & $1.39(1.15,1.67)$ \\
\hline Cohabiting & 8,554 & $20.91(19.69,22.19)$ & 3,371 & $25.09(23.13,27.16)$ & 5,183 & $17.81(16.14,19.62)$ \\
\hline Married & 35,147 & $70.66(69.20,72.07)$ & 9,412 & $63.74(61.54,65.88)$ & 25,735 & $75.79(73.69,77.77)$ \\
\hline Widowed, separated, or divorced & 2,549 & $6.34(5.69,7.06)$ & 1,025 & $8.13(6.89,9.56)$ & 1,524 & $5.01(4.36,5.75)$ \\
\hline \multicolumn{7}{|l|}{ Mother's education } \\
\hline Primary or lower & 14,581 & $26.79(25.01,28.64)$ & 2,840 & $19.10(16.23,22.33)$ & 11,741 & $32.50(30.13,34.97)$ \\
\hline Secondary & 21,534 & $47.77(46.05,49.49)$ & 7,051 & $50.38(47.69,53.07)$ & 14,483 & $45.84(43.67,48.02)$ \\
\hline Higher & 11,124 & $25.43(23.55,27.42)$ & 4,349 & $30.52(27.94,33.23)$ & 6,775 & $21.66(18.84,24.78)$ \\
\hline \multicolumn{7}{|l|}{ Partner's education } \\
\hline Primary or lower & 17,089 & $31.37(29.25,33.57)$ & 3,170 & $23.67(20.41,27.28)$ & 13,919 & $41.72(38.40,45.12)$ \\
\hline Secondary & 17,158 & $37.33(35.82,38.86)$ & 5,588 & $43.97(41.16,46.83)$ & 11,570 & $38.51(36.46,40.61)$ \\
\hline Higher & 9,443 & $22.86(20.95,24.88)$ & 4,020 & $32.35(29.64,35.19)$ & 5,423 & $19.76(16.58,23.38)$ \\
\hline Employed & 29,047 & $60.28(58.63,61.92)$ & 8,550 & $59.57(57.03,62.06)$ & 20,497 & $60.94(58.60,63.22)$ \\
\hline \multicolumn{7}{|l|}{ Wealth index } \\
\hline Poorest & 15,864 & $25.76(23.90,27.70)$ & 2,119 & $12.93(10.43,15.92)$ & 13,745 & $35.27(32.22,38.45)$ \\
\hline Poorer & 11,765 & $22.73(21.37,24.15)$ & 2,684 & $16.87(14.79,19.18)$ & 9,081 & $27.08(25.26,28.98)$ \\
\hline Middle & 8,264 & $18.62(17.27,20.05)$ & 3,152 & $21.85(19.67,24.20)$ & 5,112 & $16.23(14.67,17.92)$ \\
\hline Richer & 6,503 & $17.63(16.23,19.12)$ & 3,454 & $25.74(23.04,28.63)$ & 3,049 & $11.61(10.03,13.41)$ \\
\hline Richest & 4,848 & $15.26(13.31,17.45)$ & 2,831 & $22.62(19.72,25.80)$ & 2,017 & $9.81(7.10,13.40)$ \\
\hline
\end{tabular}

\section{Place of birth and related study variables across geographic strata}

In the total population surveyed by the DHS, 10,339 of 47,244 women had information on their place of delivery. Of this, $20.16 \%$ mothers gave birth at home [95\% confidence interval (Cl): 18.04, 22.45]. A greater proportion of women in the rural area delivered at home [26.16\% (95\% Cl: $23.22,29.32)]$ compared to women in urban communities [12.49\% (95\% Cl: 9.68, 15.96)]. For our analytical sample of 3,084 women aged 15 to 49 years old with one birth in the preceding year of the survey and who were married or cohabiting, 743 women [18.97\% $(95 \%$ Cl: 16.04, 22.28)] 
gave birth at home while 2,341 [81.03\% (95\% Cl: 77.72, 83.96)] women had an institutional delivery. More women in rural areas delivered at home $[22.95 \%(95 \% \mathrm{Cl}: 18.93,27.54)]$ than their counterparts in urban areas $[13.49 \%(95 \% \mathrm{Cl}: 9.59,18.66)]$, reflecting a significant difference between home delivery prevalence among women relative to their place of residence.

Our study consistently found a higher home delivery prevalence for women with and whose partners had at least a secondary education in the overall analytical sample, as well as in the urban and rural groups. Home delivery prevalence is highest among women who are unemployed and who belong to the poorest wealth category. Home delivery is more prevalent among rural women who are married [72.37\% (95\% Cl: 63.98 , 79.43)] and make their own healthcare decisions [48.88\% (95\% Cl: $41.43,56.38)]$, compared to their overall Philippine and rural counterparts. A considerable proportion of urban and rural women still delivered at home despite having adequate ANC visits. Rural women who have an emergency preparedness plan during pregnancy also reported a higher proportion of home delivery compared to their counterparts in the Philippine and urban population [69.10\% (95\% Cl: 56.61, 79.31)] [Table 3]. 
Table 3

Prevalence of home delivery by study factors among women aged 15-49 years with husbands/partners and one birth in the year preceding the survey

\begin{tabular}{|c|c|c|c|c|c|c|c|c|c|}
\hline \multirow[t]{2}{*}{ Variable } & \multicolumn{3}{|c|}{ Philippines } & \multicolumn{3}{|c|}{ Urban } & \multicolumn{3}{|l|}{ Rural } \\
\hline & $\mathbf{N}^{\mathrm{a}}$ & $\begin{array}{l}\text { \% home delivery } \\
(95 \% \mathrm{Cl})\end{array}$ & $\begin{array}{l}\text { p- } \\
\text { value }\end{array}$ & $\mathbf{N}^{\mathrm{a}}$ & $\begin{array}{l}\% \text { home } \\
\text { delivery ( } 95 \% \\
\text { Cl) }\end{array}$ & $\begin{array}{l}\mathrm{p}- \\
\text { value }\end{array}$ & $\mathbf{N}^{\mathrm{a}}$ & $\begin{array}{l}\text { \% home } \\
\text { delivery ( } 95 \% \\
\mathrm{Cl})\end{array}$ & $\begin{array}{l}\mathrm{p}- \\
\text { value }\end{array}$ \\
\hline \multicolumn{10}{|l|}{ Place of delivery } \\
\hline Home & 743 & $\begin{array}{l}18.97(16.04 \\
22.28)\end{array}$ & - & 122 & $\begin{array}{l}13.49(9.58 \\
18.67)\end{array}$ & - & 621 & $\begin{array}{l}22.95(18.93 \\
27.54)\end{array}$ & - \\
\hline Institutional & 2,341 & $\begin{array}{l}81.03(77.72, \\
83.96)\end{array}$ & - & 776 & $\begin{array}{l}86.51(81.33 \\
90.42)\end{array}$ & - & 1,565 & $\begin{array}{l}77.05(72.46, \\
81.07)\end{array}$ & - \\
\hline \multicolumn{10}{|l|}{ Socio-economic factors } \\
\hline \multicolumn{3}{|l|}{ Mother's education } & \multirow{4}{*}{$\begin{array}{l}< \\
0.01\end{array}$} & & & \multirow{4}{*}{$\begin{array}{l}< \\
0.01\end{array}$} & & & \multirow{4}{*}{$\begin{array}{l}< \\
0.01\end{array}$} \\
\hline Primary or lower & 380 & $\begin{array}{l}49.44(40.27 \\
58.65)\end{array}$ & & 59 & $\begin{array}{l}52.36(33.29 \\
70.78)\end{array}$ & & 321 & $\begin{array}{l}48.19(38.22 \\
58.30)\end{array}$ & \\
\hline Secondary & 311 & $\begin{array}{l}49.56(45.84 \\
53.28)\end{array}$ & & 53 & $\begin{array}{l}42.70(25.87 \\
61.42)\end{array}$ & & 1,036 & $\begin{array}{l}47.75(38.20 \\
57.46)\end{array}$ & \\
\hline Higher & 52 & $\begin{array}{l}28.75(25.37 \\
32.39)\end{array}$ & & 10 & $\begin{array}{l}4.93(1.88, \\
12.35)\end{array}$ & & 516 & $\begin{array}{l}4.07(2.35 \\
6.94)\end{array}$ & \\
\hline \multicolumn{3}{|l|}{ Partner's education } & \multirow{4}{*}{$\begin{array}{l}< \\
0.01\end{array}$} & & & \multirow{4}{*}{$\begin{array}{l}< \\
0.01\end{array}$} & & & \multirow{4}{*}{$\begin{array}{l}< \\
0.01\end{array}$} \\
\hline Primary or lower & 468 & $\begin{array}{l}58.94(50.54 \\
66.84)\end{array}$ & & 60 & $\begin{array}{l}49.33(30.13 \\
68.73)\end{array}$ & & 408 & $\begin{array}{l}63.05(54.97 \\
70.45)\end{array}$ & \\
\hline Secondary & 232 & $\begin{array}{l}35.96(28.85 \\
43.75)\end{array}$ & & 47 & $\begin{array}{l}38.92(23.13 \\
57.44)\end{array}$ & & 185 & $\begin{array}{l}34.70(27.58 \\
42.57)\end{array}$ & \\
\hline Higher & 43 & $\begin{array}{l}5.10(2.36 \\
10.69)\end{array}$ & & 15 & $\begin{array}{l}11.75(3.98 \\
29.98)\end{array}$ & & 28 & $\begin{array}{l}2.26(1.29 \\
3.91)\end{array}$ & \\
\hline \multicolumn{3}{|l|}{ Mother's employment status } & \multirow[t]{3}{*}{0.11} & & & \multirow[t]{3}{*}{0.60} & & & \multirow{3}{*}{$\begin{array}{l}< \\
0.01\end{array}$} \\
\hline Unemployed & 533 & $\begin{array}{l}70.61(63.33 \\
76.96)\end{array}$ & & 78 & $\begin{array}{l}58.65 \text { (42.98, } \\
72.75)\end{array}$ & & 455 & $\begin{array}{l}75.78 \text { (68.96, } \\
81.51)\end{array}$ & \\
\hline Employed & 207 & $\begin{array}{l}29.33(22.98 \\
36.61)\end{array}$ & & 44 & $\begin{array}{l}41.35(27.25 \\
57.02)\end{array}$ & & 163 & $\begin{array}{l}24.22(18.49 \\
31.04)\end{array}$ & \\
\hline \multicolumn{3}{|l|}{ Wealth index } & \multirow{4}{*}{$\begin{array}{l}< \\
0.01\end{array}$} & & & \multirow{4}{*}{$\begin{array}{l}< \\
0.01\end{array}$} & & & \multirow{4}{*}{$\begin{array}{l}< \\
0.01\end{array}$} \\
\hline Poor & 672 & $\begin{array}{l}85.13(78.03 \\
90.22)\end{array}$ & & 107 & $\begin{array}{l}88.18(71.34 \\
95.72)\end{array}$ & & 543 & $\begin{array}{l}84.58(76.71, \\
90.14)\end{array}$ & \\
\hline Middle & 48 & $\begin{array}{l}11.12(6.78 \\
17.70)\end{array}$ & & 11 & $\begin{array}{l}7.38(2.32, \\
21.13)\end{array}$ & & 48 & $\begin{array}{l}9.11(4.74, \\
16.78)\end{array}$ & \\
\hline Rich & 23 & $3.75(1.62,8.47)$ & & 4 & $\begin{array}{l}4.43(0.68 \\
23.82)\end{array}$ & & 30 & $\begin{array}{l}6.31(3.45 \\
11.18)\end{array}$ & \\
\hline \multicolumn{10}{|l|}{ Individual factors } \\
\hline Age & - & - & 0.28 & - & - & 0.24 & - & - & 0.09 \\
\hline Marital status & & & \multirow[t]{3}{*}{0.02} & & & 0.81 & & & \multirow[t]{3}{*}{0.01} \\
\hline Cohabiting & 200 & $\begin{array}{l}35.65(28.61, \\
43.38)\end{array}$ & & 57 & $\begin{array}{l}54.42(38.57 \\
69.42)\end{array}$ & & 143 & $\begin{array}{l}27.63(20.57 \\
36.02)\end{array}$ & \\
\hline Married & 543 & $\begin{array}{l}64.35(56.62, \\
71.39)\end{array}$ & & 65 & $\begin{array}{l}45.58(30.58 \\
61.43)\end{array}$ & & 478 & $\begin{array}{l}72.37 \text { (63.98, } \\
79.43)\end{array}$ & \\
\hline \multicolumn{10}{|l|}{ Pregnancy-related factors } \\
\hline Parity & & & \multicolumn{3}{|l|}{$<.01$} & \multicolumn{3}{|l|}{$\begin{array}{l}< \\
0.01\end{array}$} & $\begin{array}{l}<.01 \\
0.01\end{array}$ \\
\hline
\end{tabular}




\begin{tabular}{|c|c|c|c|c|c|c|c|c|c|}
\hline \multirow[t]{2}{*}{ Variable } & \multicolumn{3}{|c|}{ Philippines } & \multicolumn{3}{|c|}{ Urban } & \multicolumn{3}{|c|}{ Rural } \\
\hline & $\mathbf{N}^{\mathbf{a}}$ & $\begin{array}{l}\text { \% home delivery } \\
(95 \% \mathrm{Cl})\end{array}$ & $\begin{array}{l}\mathrm{p}- \\
\text { value }\end{array}$ & $\mathrm{N}^{\mathrm{a}}$ & $\begin{array}{l}\text { \% home } \\
\text { delivery (95\% } \\
\text { Cl) }\end{array}$ & $\begin{array}{l}\text { p- } \\
\text { value }\end{array}$ & $\mathbf{N}^{\mathbf{a}}$ & $\begin{array}{l}\% \text { home } \\
\text { delivery (95\% } \\
\text { Cl) }\end{array}$ & $\begin{array}{l}\mathrm{p}- \\
\text { value }\end{array}$ \\
\hline Para one & 44 & $4.63(3.05,6.98)$ & & 7 & $\begin{array}{l}3.20(1.23 \\
8.07)\end{array}$ & & 37 & $\begin{array}{l}5.25(3.32 \\
8.20)\end{array}$ & \\
\hline Para 2-4 & 425 & $\begin{array}{l}60.74(53.84 \\
67.24)\end{array}$ & & 70 & $\begin{array}{l}64.00(48.66 \\
76.93)\end{array}$ & & 355 & $\begin{array}{l}59.35(51.81 \\
66.47)\end{array}$ & \\
\hline Para 5 or more & 274 & $\begin{array}{l}34.63(28.16 \\
41.72)\end{array}$ & & 45 & $\begin{array}{l}32.80(20.20 \\
48.49)\end{array}$ & & 229 & $\begin{array}{l}35.41(28.29 \\
43.24)\end{array}$ & \\
\hline Birth order & - & - & $\begin{array}{l}< \\
0.01\end{array}$ & - & - & & - & - & $\begin{array}{l}< \\
0.01\end{array}$ \\
\hline $\begin{array}{l}\text { Women's decision-making } \\
\text { power }\end{array}$ & & & 0.05 & & & $\dot{0} 01$ & & & 0.29 \\
\hline Respondent alone & 340 & $\begin{array}{l}48.88(41.43, \\
56.38)\end{array}$ & & 46 & $\begin{array}{l}35.46(23.25 \\
49.92)\end{array}$ & & 294 & $\begin{array}{l}54.62(46.40 \\
62.60)\end{array}$ & \\
\hline $\begin{array}{l}\text { Both respondent and } \\
\text { husband/partner }\end{array}$ & 358 & $\begin{array}{l}43.74(36.68, \\
51.06)\end{array}$ & & 63 & $\begin{array}{l}51.48(36.43 \\
66.26)\end{array}$ & & 295 & $\begin{array}{l}40.44(32.83 \\
48.53)\end{array}$ & \\
\hline $\begin{array}{l}\text { Husband/partner, or someone } \\
\text { else }\end{array}$ & 45 & $\begin{array}{l}7.38(4.16 \\
12.76)\end{array}$ & & 13 & $\begin{array}{l}13.06(6.03 \\
26.02)\end{array}$ & & 32 & $\begin{array}{l}4.95(2.37 \\
10.02)\end{array}$ & \\
\hline ANC visits & & & $<01$ & & & $<01$ & & & $<01$ \\
\hline $\begin{array}{l}\text { Inadequate ANC visits (less } \\
\text { than 4) }\end{array}$ & 150 & $\begin{array}{l}18.63(14.35, \\
23.83)\end{array}$ & & 18 & $\begin{array}{l}41.63(20.53 \\
66.32)\end{array}$ & & 132 & $\begin{array}{l}41.07(30.81 \\
52.17)\end{array}$ & \\
\hline $\begin{array}{l}\text { Adequate ANC visits (at least } \\
\text { 4) }\end{array}$ & 189 & $\begin{array}{l}26.56(21.47 \\
32.36)\end{array}$ & & 36 & $\begin{array}{l}58.37(33.68, \\
79.47)\end{array}$ & & 153 & $\begin{array}{l}58.93(47.83 \\
69.19)\end{array}$ & \\
\hline $\begin{array}{l}\text { Emergency preparedness } \\
\text { during pregnancy }\end{array}$ & & & $\begin{array}{l}<.01 \\
0.01\end{array}$ & & & 0.36 & & & $\begin{array}{l}<.01 \\
0.01\end{array}$ \\
\hline Not prepared & 88 & $\begin{array}{l}12.34(8.60 \\
17.41)\end{array}$ & & 45 & $\begin{array}{l}81.83(62.09 \\
92.53)\end{array}$ & & 79 & $\begin{array}{l}30.90(20.69 \\
43.39)\end{array}$ & \\
\hline Prepared & 251 & $\begin{array}{l}32.85(27.94 \\
38.16)\end{array}$ & & 9 & $\begin{array}{l}18.17(7.47 \\
37.91)\end{array}$ & & 206 & $\begin{array}{l}69.10(56.61, \\
79.31)\end{array}$ & \\
\hline
\end{tabular}

\section{Determinants of home delivery in the Philippines}

The weighted univariate logistic regression analysis showed that home delivery is negatively associated with cohabiting and making healthcare decisions with their husband or partner, and positively associated with all other determinants in the unadjusted model as shown in Table 4. The weighted multivariable logistic regression analysis indicates that the odds of home delivery is $7 \%$ less with each year increase in age [Adjusted Odds Ratio (aOR): 0.93 (95\% Cl: 0.89, 0.98)] while each increase in birth order results in a $15 \%$ increase in the odds of home delivery [aOR: $1.15(95 \% \mathrm{Cl}: 0.94,1.40)$ ], although evidence for birth order is weak $\left(P_{\text {age }}=<0.01\right.$ vs. $\left.P_{\text {birth_order }}=0.16\right)$. For education of mothers and their partners, the observed relationship is similar with an increasing trend in the odds of home delivery from secondary to primary education or lower. We observe similar positive trends for home delivery for wealth index with those categorized as middle-income class having 1.91 times higher odds [aOR: 1.91 (95\% Cl: 0.60, 6.07)], and those categorized as poor having 3.45 higher odds of home delivery [aOR: $3.45(95 \% \mathrm{Cl}: 1.29,9.20)$ ], although evidence for the former is weak $(P=0.27)$. A similar increasing trend is observed for parity with those having two to four [aOR: 2.32 (95\% Cl: 1.23, 4.36)] and five or more children [aOR: 4.50 (95\% Cl: 1.42, 14.27)] being more likely to deliver at home compared to those with only one child, with strong evidence for both $(P=<0.01$ and $P=0.01)$. Women whose decision-making power depends on other individuals but themselves are more likely to have a home delivery by 1.55 times $(95 \% \mathrm{Cl}: 0.80,2.99)$ compared to those who decide for themselves, although the evidence for this association is weak $(P=0.19)$. Inadequate antenatal care visits and having no emergency preparedness plan also increase the odds of the mother giving birth at home [aOR: 3.29 (95\% Cl: 2.18, 4.95); aOR: 1.20 (95\% Cl: $0.74,1.93)]$. 
Table 4

Determinants of home delivery in the overall population of 15-to-49-year-old women with husbands/partners and one birth in the year preceding the survey

\begin{tabular}{|c|c|c|c|c|}
\hline Variable & Crude OR (95\% Cl) & p-value & Adjusted OR $(95 \% \mathrm{Cl})^{\mathrm{a}}$ & p-value \\
\hline Age & $1.00(0.97,1.03)$ & 0.90 & $0.93(0.89,0.98)$ & $<0.01$ \\
\hline \multicolumn{5}{|l|}{ Marital status } \\
\hline Married & 1.00 & - & 1.00 & - \\
\hline Cohabiting & $0.70(0.47,1.04)$ & 0.08 & $0.77(0.50,1.18)$ & 0.23 \\
\hline \multicolumn{5}{|l|}{ Mother's education } \\
\hline Higher & 1.00 & - & 1.00 & - \\
\hline Secondary & $7.19(3.84,13.45)$ & $<0.01$ & $2.38(1.15,4.92)$ & 0.02 \\
\hline Primary or lower & $34.90(17.66,69.00)$ & $<0.01$ & $4.55(2.08,9.96)$ & $<0.01$ \\
\hline \multicolumn{5}{|l|}{ Partner's education } \\
\hline Higher & 1.00 & - & 1.00 & - \\
\hline Secondary & $3.88(1.72,8.77)$ & $<0.01$ & $1.53(0.69,3.38)$ & 0.30 \\
\hline Primary or lower & $17.49(7.34,41.70)$ & $<0.01$ & $2.74(1.13,6.61)$ & 0.03 \\
\hline \multicolumn{5}{|l|}{ Wealth index } \\
\hline Rich & 1.00 & - & 1.00 & - \\
\hline Middle & $4.89(1.75,13.63)$ & $<0.01$ & $1.91(0.60,6.07)$ & 0.27 \\
\hline Poor & $19.51(7.87,48.33)$ & $<0.01$ & $3.45(1.29,9.20)$ & 0.01 \\
\hline \multicolumn{5}{|l|}{ Parity } \\
\hline Para one & 1.00 & - & 1.00 & - \\
\hline Para 2-4 & $3.10(1.91,5.05)$ & $<0.01$ & $2.32(1.23,4.36)$ & 0.01 \\
\hline Para 5 or more & $11.45(6.37,20.59)$ & $<0.01$ & $4.50(1.42,14.27)$ & 0.01 \\
\hline Birth order & $1.39(1.28,1.50)$ & $<0.01$ & $1.15(0.94,1.40)$ & 0.16 \\
\hline \multicolumn{5}{|l|}{ Women's decision-making power } \\
\hline Respondent alone & 1.00 & - & 1.00 & - \\
\hline Both respondent and husband/partner & $0.85(0.60,1.20)$ & 0.36 & $0.92(0.62,1.36)$ & 0.69 \\
\hline Husband/partner, or someone else & $2.18(1.18,4.03)$ & 0.01 & $1.55(0.80,2.99)$ & 0.19 \\
\hline \multicolumn{5}{|l|}{ ANC visits } \\
\hline Adequate ANC visits (at least 4) & 1.00 & - & 1.00 & - \\
\hline Inadequate ANC visits (less than 4) & $6.68(4.23,10.54)$ & $<0.01$ & $3.29(2.18,4.95)$ & $<0.01$ \\
\hline \multicolumn{5}{|l|}{ Emergency preparedness during pregnancy } \\
\hline Prepared & 1.00 & - & 1.00 & - \\
\hline Not prepared & $2.94(1.75,4.95)$ & $<0.01$ & $1.20(0.74,1.93)$ & 0.46 \\
\hline
\end{tabular}

\section{Determinants of home delivery for urban and rural communities in the Philippines}

The weighted univariate logistic regression analysis presented in Table 5 shows that home delivery is negatively associated with cohabiting and making healthcare decisions with their husband or partner for only the rural population although evidence is weak. Home delivery is 
positively associated with all other determinants in the unadjusted models in both populations. The weighted multivariable logistic regression analyses show that the odds of home delivery decreased by $11 \%$ and $5 \%$ for each year increase in age among urban and rural women, respectively [Urban aOR: 0.89 (95\% $\mathrm{Cl}$ : $0.80,0.99)$; Rural aOR: 0.95 (95\% $\mathrm{Cl}: 0.91,1.00)$ ]. Among urban mothers, the odds of home delivery is 1.19 times more $(95 \% \mathrm{Cl}: 0.73,1.95)$ for each increase in birth order with evidence for this being weak $(P=0.48)$ with a similar association observed for rural women [aOR: $1.14(95 \% \mathrm{Cl}$ : 0.92, 1.41)]. Among urban women, a negative association is only observed for not having emergency preparedness plans during pregnancy [aOR: $0.42(95 \% \mathrm{Cl}: 0.15,1.20)$ ] with evidence being weak $(P=0.11)$. For rural women, a negative association is observed for cohabiting [aOR: 0.77 (95\% Cl: $0.47,1.25)$ ], being in the middle-income category [aOR: 0.70 (95\% Cl: 0.23 , 2.11)], and making decisions with her husband or partner [aOR: 0.87 (95\% $\mathrm{Cl}: 0.57,1.35)$ ]. Lower education of mothers and their partners increased the odds of a home delivery. There is an increasing trend in home delivery favoring the poorest wealth category with an increased odds of home delivery compared to those in the richest category [Urban aOR: 5.63 (95\% Cl: 0.77, 41.14); Rural aOR: 1.71 (95\% Cl: 0.78, 3.77)]. Evidence for this association with wealth index is weak for both populations $\left(P_{\text {urban }}=0.09 ; P_{\text {rural }}=0.18\right)$. This positive association is similarly observed for parity, with those having five or more children having the highest odds of home delivery for both urban and rural populations although evidence is weak for rural women. Compared to the overall population, rural women with inadequate ANC visits have a higher odds of giving birth at home [aOR: 3.79 (95\% Cl: $2.43,5.91)$ ], but this association is attenuated among urban women [aOR: 1.85 ( $95 \%$ Cl: 0.69 , 4.92)]. 
Table 5

Determinants of home delivery among 15-to-49-year-old women with husbands/partners and one birth in the year preceding the survey in urban and rural communities

\begin{tabular}{|c|c|c|c|c|c|c|c|c|}
\hline & Urban & & & & Rural & & & \\
\hline Variable & $\begin{array}{l}\text { Crude OR (95\% } \\
\mathrm{Cl})\end{array}$ & $\begin{array}{l}\text { p- } \\
\text { value }\end{array}$ & $\begin{array}{l}\text { Adjusted OR } \\
(95 \% \mathrm{Cl})^{\mathrm{a}}\end{array}$ & $\begin{array}{l}\mathrm{p}- \\
\text { value }\end{array}$ & $\begin{array}{l}\text { Crude OR } \\
(95 \% \mathrm{Cl})\end{array}$ & $\begin{array}{l}\mathrm{p}- \\
\text { value }\end{array}$ & $\begin{array}{l}\text { Adjusted OR } \\
(95 \% \mathrm{Cl})^{a}\end{array}$ & $\begin{array}{l}\mathrm{p}- \\
\text { value }\end{array}$ \\
\hline Age & $\begin{array}{l}0.97(0.92 \\
1.02)\end{array}$ & 0.26 & $\begin{array}{l}0.89(0.80 \\
0.99)\end{array}$ & 0.03 & $\begin{array}{l}1.01(0.97 \\
1.05)\end{array}$ & 0.60 & $\begin{array}{l}0.95(0.91 \\
1.00)\end{array}$ & 0.05 \\
\hline \multicolumn{9}{|l|}{ Marital status } \\
\hline Married & 1.00 & - & 1.00 & - & 1.00 & - & 1.00 & - \\
\hline Cohabiting & $\begin{array}{l}1.20(0.54 \\
2.67)\end{array}$ & 0.67 & $\begin{array}{l}1.03(0.0 .44 \\
2.39)\end{array}$ & 0.95 & $\begin{array}{l}0.62(0.39 . \\
1.00)\end{array}$ & 0.05 & $\begin{array}{l}0.77(0.47 \\
1.25)\end{array}$ & 0.29 \\
\hline \multicolumn{9}{|l|}{ Mother's education } \\
\hline Higher & 1.00 & - & 1.00 & - & 1.00 & - & 1.00 & - \\
\hline Secondary & $\begin{array}{l}4.77(1.63 \\
14.01)\end{array}$ & $<.01$ & $\begin{array}{l}1.43(0.36 \\
5.72)\end{array}$ & 0.61 & $\begin{array}{l}8.26(3.86 \\
17.68)\end{array}$ & $<.01$ & $\begin{array}{l}3.10(1.26 \\
7.62)\end{array}$ & 0.01 \\
\hline Primary or lower & $\begin{array}{l}39.62(11.40 \\
137.67)\end{array}$ & $\begin{array}{l}< \\
0.01\end{array}$ & $\begin{array}{l}4.87(0.94 \\
25.31)\end{array}$ & 0.06 & $\begin{array}{l}30.69 \text { (13.63, } \\
69.08)\end{array}$ & $\begin{array}{l}< \\
0.01\end{array}$ & $\begin{array}{l}4.32(1.76, \\
10.58)\end{array}$ & $\begin{array}{l}< \\
0.01\end{array}$ \\
\hline \multicolumn{9}{|l|}{ Partner's education } \\
\hline Higher & 1.00 & - & 1.00 & - & 1.00 & - & 1.00 & - \\
\hline Secondary & $\begin{array}{l}1.96(0.62, \\
6.23)\end{array}$ & 0.26 & $\begin{array}{l}1.06(0.31 \\
3.69)\end{array}$ & 0.92 & $\begin{array}{l}7.71(3.31 \\
17.98)\end{array}$ & $\begin{array}{l}< \\
0.01\end{array}$ & $\begin{array}{l}2.93(1.17 \\
7.37)\end{array}$ & 0.02 \\
\hline Primary or lower & $\begin{array}{l}9.93(2.56, \\
38.54)\end{array}$ & $\begin{array}{l}< \\
0.01\end{array}$ & $\begin{array}{l}1.95(0.55 \\
6.97)\end{array}$ & 0.30 & $\begin{array}{l}29.95(12.25 \\
73.22)\end{array}$ & $\begin{array}{l}< \\
0.01\end{array}$ & $\begin{array}{l}5.88(2.18 \\
15.86)\end{array}$ & $\begin{array}{l}< \\
0.01\end{array}$ \\
\hline \multicolumn{9}{|l|}{ Wealth index } \\
\hline Rich & 1.00 & - & 1.00 & - & 1.00 & - & 1.00 & - \\
\hline Middle & $\begin{array}{l}3.03(0.29 \\
31.67)\end{array}$ & 0.35 & $\begin{array}{l}1.73(0.17 \\
18.11)\end{array}$ & 0.65 & $\begin{array}{l}2.00(0.70 \\
5.68)\end{array}$ & 0.20 & $\begin{array}{l}0.70(0.23 \\
2.11)\end{array}$ & 0.52 \\
\hline Poor & $\begin{array}{l}20.99(2.62, \\
168.00)\end{array}$ & $\begin{array}{l}< \\
0.01\end{array}$ & $\begin{array}{l}5.63(0.77 \\
41.14)\end{array}$ & 0.09 & $\begin{array}{l}10.07(4.46 \\
22.75)\end{array}$ & $\begin{array}{l}< \\
0.01\end{array}$ & $\begin{array}{l}1.71(0.78 \\
3.77)\end{array}$ & 0.18 \\
\hline \multicolumn{9}{|l|}{ Parity } \\
\hline Para one & 1.00 & - & 1.00 & - & 1.00 & - & 1.00 & - \\
\hline Para 2-4 & $\begin{array}{l}4.28(1.46, \\
12.52)\end{array}$ & 0.01 & $\begin{array}{l}4.44(1.02, \\
19.39)\end{array}$ & 0.05 & $\begin{array}{l}2.84(1.67 \\
4.85)\end{array}$ & $\begin{array}{l}< \\
0.01\end{array}$ & $\begin{array}{l}1.97(0.96 \\
4.05)\end{array}$ & 0.07 \\
\hline Para 5 or more & $\begin{array}{l}27.87(7.92, \\
98.06)\end{array}$ & $\begin{array}{l}< \\
0.01\end{array}$ & $\begin{array}{l}18.81(1.21 \\
292.23)\end{array}$ & 0.04 & $\begin{array}{l}7.61(3.96, \\
14.63)\end{array}$ & $\begin{array}{l}< \\
0.01\end{array}$ & $\begin{array}{l}2.52(0.67 \\
9.45)\end{array}$ & 0.17 \\
\hline Birth order & $\begin{array}{l}1.56(1.31 \\
1.85)\end{array}$ & $\begin{array}{l}< \\
0.01\end{array}$ & $\begin{array}{l}1.19(0.73 \\
1.95)\end{array}$ & 0.48 & $\begin{array}{l}1.31(1.20 \\
1.43)\end{array}$ & $\begin{array}{l}< \\
0.01\end{array}$ & $\begin{array}{l}1.14(0.92, \\
1.41)\end{array}$ & 0.22 \\
\hline \multicolumn{9}{|l|}{$\begin{array}{l}\text { Women's decision-making } \\
\text { power }\end{array}$} \\
\hline Respondent alone & 1.00 & - & 1.00 & - & 1.00 & - & 1.00 & - \\
\hline $\begin{array}{l}\text { Both respondent and } \\
\text { husband/partner }\end{array}$ & $\begin{array}{l}1.06(0.50 \\
2.23)\end{array}$ & 0.88 & $\begin{array}{l}1.11(0.45 \\
2.71)\end{array}$ & 0.82 & $\begin{array}{l}0.78(0.53 \\
1.15)\end{array}$ & 0.21 & $\begin{array}{l}0.87(0.57 \\
1.35)\end{array}$ & 0.54 \\
\hline $\begin{array}{l}\text { Husband/partner, or someone } \\
\text { else }\end{array}$ & $\begin{array}{l}4.26(1.82 \\
9.94)\end{array}$ & $<.01$ & $\begin{array}{l}2.02(0.66 \\
6.17)\end{array}$ & 0.22 & $\begin{array}{l}1.53(0.66 \\
3.55)\end{array}$ & 0.32 & $\begin{array}{l}1.41(0.56 \\
3.56)\end{array}$ & 0.47 \\
\hline
\end{tabular}

a Weighted multivariable logistic regression analysis, with place of delivery (health facility or home) as the dependent variable, and all variables in this table as independent variables for all models. Age and birth order were modeled as continuous variables. 


\begin{tabular}{|c|c|c|c|c|c|c|c|c|}
\hline & Urban & & & & Rural & & & \\
\hline $\begin{array}{l}\text { Adequate ANC visits (at least } \\
\text { 4) }\end{array}$ & 1.00 & - & 1.00 & - & 1.00 & - & 1.00 & - \\
\hline $\begin{array}{l}\text { Inadequate ANC visits (less } \\
\text { than 4) }\end{array}$ & $\begin{array}{l}7.55(2.73 \\
20.92)\end{array}$ & $\begin{array}{l}< \\
0.01\end{array}$ & $\begin{array}{l}1.85(0.69 \\
4.92)\end{array}$ & 0.22 & $\begin{array}{l}6.13(3.72 \\
10.10)\end{array}$ & $\begin{array}{l}< \\
0.01\end{array}$ & $\begin{array}{l}3.79(2.43 \\
5.91)\end{array}$ & $\begin{array}{l}< \\
0.01\end{array}$ \\
\hline \multicolumn{9}{|l|}{$\begin{array}{l}\text { Emergency preparedness } \\
\text { during pregnancy }\end{array}$} \\
\hline Prepared & 1.00 & - & 1.00 & - & 1.00 & - & 1.00 & - \\
\hline Not prepared & $\begin{array}{l}1.64(0.57 \\
4.74)\end{array}$ & 0.36 & $\begin{array}{l}0.42(0.15 \\
1.20)\end{array}$ & 0.11 & $\begin{array}{l}3.68(2.03 \\
6.67)\end{array}$ & $<.01$ & $\begin{array}{l}1.80(1.08 \\
3.02)\end{array}$ & 0.03 \\
\hline
\end{tabular}

\section{Discussion}

Our study showed that $20.16 \%$ of 47,244 women aged $15-49$ years in the Philippines delivered at home. This proportion was higher than what was reported among urban mothers $(12.49 \%)$ but lower than what was reported in the rural communities $(26.16 \%)$. This has been the lowest proportion of home deliveries since the 1993 NDHS [26] and mirrors the improvements made in reducing the infant mortality rate, under-5 mortality rate, and maternal mortality rate in the country $[1,9]$. Despite this progress, surveys and the present analysis continue to show that women still deliver at home where there is low possibility of being assisted by skilled birth attendants. More importantly, delivering at home could delay life-saving treatment should complications due to childbirth occur [8].

There are a number of factors that influence the choice of women to deliver at home, including those identified by our study: age, marital status, mother's education, husband's or partner's education, wealth index, parity, birth order, women's decision-making power, ANC visits, and emergency preparedness. Our findings suggest these factors yield similar results among the overall, urban, and rural populations, but with a relatively greater effect observed for the rural population in most of the study factors considered similar to the findings of a previous study [38]. The present study further highlights existing disparities between women in the rural communities with more home deliveries despite comparable proportions of rural to urban women on key variables such as having adequate ANC visits and being prepared financially during pregnancy for emergencies. These likely reflect the inequalities associated with health service delivery, specifically in the distribution of health professionals and low investments in the health sector infrastructure [22]. A local study on the trends in neonatal mortality and child health inequality used facility-based delivery as a proxy of health service delivery, and determined that it is an effective but complex intervention that requires a fully functional system [22]. The government has since made efforts to make healthcare services more accessible through the MNCHN Strategy and the newly passed Universal Health Care Act $[9,11]$. Our study also identified wealth through assets and wealth proxied by having emergency funds during pregnancy as important factors influencing the choice of home delivery. The odds of home delivery is most pronounced in the poorest wealth category with a slightly larger effect among urban women. The observed association between home delivery and wealth index is consistent with the results of other studies [28,31,34,35]. Women of low economic status are likely to choose birth at home because of associated costs with health facility delivery [35]. The National Health Insurance Program (NHIP) covers health expenses during antenatal period and delivery of women. However, there are other costs related to pregnancy and delivery that need to be considered and acknowledged when interpreting our findings and when advocating for institutional delivery. In our analyses, we found that lack of emergency funds during pregnancy resulted in an increased likelihood for home delivery among rural women, but found the reverse for urban women. Especially in rural areas and geographically isolated and disadvantaged areas (GIDA), transportation costs to the facility may be expensive as health facilities usually require long-distance travels that could discourage women from seeking care at these facilities. The present analysis was unable to account for time and distance for travel, but this theory on distance being associated with place of delivery has been refuted by a local study, with most home deliveries being close to health facilities [36]. A geospatial analysis in Indonesia supports this finding that area of residence is not associated with place of delivery [39]. Increasing health insurance coverage is likely effective in increasing facility-based deliveries [23], but we also need to account for cultural context and maternal satisfaction when we interpret findings on this topic and make recommendations for policy $[24,36,40]$.

Women's decision-making power in healthcare also influences the choice of place of delivery. We found that women in both urban and rural settings whose healthcare decisions were based on their husband, partner, or someone else were more likely to give birth at home. This is consistent with previous reports that there is a negative effect on the use of institutional services when women themselves are not the ultimate decision maker [28, 41-43]. This observed relationship however is more complex as others have documented an increase in home deliveries among women who make decisions for themselves $[21,44]$. Additionally, in our study population, there were more married women than those 
living with their partners, and we found cohabiting decreases the odds of giving birth at home among rural women but not among urban women although evidence is weak. This may be related to empowerment of women in making decisions as more women living with their partners made healthcare decisions on their own. However, we were not able to account for the beliefs of the women and their partners that could influence the association and could explain the slightly higher odds for home delivery observed among urban women. Also related is education, which is sometimes used as proxy measures for women's autonomy [42]. Most women and their partners in our study received a secondary education or lower with an increased odds of home delivery among those with primary or lower education relative to those with higher education. The association is attenuated among rural women. Most studies on pregnancy and delivery study the role of education in influencing women's healthcare decisions [21, 27, 28, 30-32, 34-36, 41]. Possible explanations that support our results include educated individuals having better access to health service information and having the ability to evaluate and apply such information, making them more health literate and thus more likely to seek care during pregnancy. These individuals are also likely to belong in richer wealth categories, allowing them to access quality health services better and with greater ease. Women who belong in poorer wealth categories in our study also had fewer ANC visits, and having inadequate ANC visits increases the odds of home delivery among urban and rural women. Our findings on decision-making power, the role of partners and networks, and the role of education highlight the importance of co-creation and patient participation in health service delivery involving not only the mother and her partner, but her parents and other family members, and close networks as well $[30,31]$. This includes developing innovative strategies that leverage social capital and networks of women such as providing incentives to traditional birth attendants whom women go to for healthcare to refer them to health facilities [45]. Beyond empowering women and making health information and services more accessible, providing women with better formal education is also as important as it influences health outcomes of both mother and child [42].

Our study showed that each increase in age of women decreased the odds of home delivery in our study. This is in conflict with other studies that reported increase in maternal age increased the odds of home delivery $[30,31]$. Previous experiences in health services may have been positive among our study population. It is also possible that these women had pregnancy complications that required an institutional delivery or that older women have greater autonomy and decision-making power. However, only decision-making power was measured in our study with complications and past experiences not measured in the NDHS. Because there is still a considerable proportion of women who deliver at a relatively young age, the reproductive health program may need to integrate and emphasize the importance of institutional deliveries. In our study, we also found that a significant proportion of women reported parity of at least two and a relatively high birth order number. The odds of home delivery increased with parity of two or more for both urban and rural women. For birth order, each increase in birth order also increased the odds of home delivery for both populations. Previous literature similarly reported that multiparity is associated with home delivery with the following possible explanations: larger family sizes demand more time and resources from the mother that could limit her ability to avail of health services, and uneventful deliveries with traditional birth attendants may lower risk perceptions of home delivery [27, 35]. Because the risk of complications increases with each additional pregnancy [46], women should be supported throughout pregnancy and delivery to encourage them to continue having institutional deliveries for all their pregnancies.

The study has methodological limitations that should be considered. Marital status in the model does not take into consideration data from women who are single, separated, widowed, or divorced. We restricted our analyses to women with partners so we could study the association between partner's education and women's decision-making power, which have been identified in the literature as important determinants of place of delivery $[19,21,27-31]$. Exclusion of information from women without partners at the time of the survey resulted to minimal loss of information with only less than $4 \%$ of single and separated, widowed, or divorced women with the outcome among the entire population of 47,244 women. Our study may also have unmeasured confounding that could affect the observed associations as our analyses relied on data collected and reported in the 2017 Philippine NDHS Births Recode. Some possible factors based on previous studies include receipt of health information during pregnancy, knowledge of danger signs, transport availability, time and distance to a health facility, past experiences, and history of complications. Despite this, we controlled for the effect of other known important factors such as wealth index, parity, birth order, ANC visits, and emergency preparedness. Fourth, if there are systemic differences in the women in the urban and rural communities relative to the women included in the study, there could be potential for selection bias. We also excluded observations due to missing data, which could also be a source of selection bias. Additionally, because MMR remains high in the country remains high [1,5,9], our study could potentially have Neyman bias[47] where those with poor maternal outcomes or those who have died due to complications during pregnancy or delivery could not be included in the survey. However, because this is a secondary analysis, we are not able to quantify the biases present arising from various sources such as non-participation. There also could be recall bias present because the information collected in the survey was based on self-reporting. However, the present analysis was restricted to women with one birth in the last year preceding the survey and no longer included information from other births from previous years minimizing potential for recall issues. Our study also focused mainly on variables that are 'static' and unchanged over time that could further minimize this problem. As typical in cross-sectional study designs, our study cannot establish a clear temporal association between the study factors and place of delivery. Despite this, our study provides updated contextual evidence on key determinants of home delivery in the Philippines, which is one of the countries that accounts for $80 \%$ of child deaths worldwide [2]. 


\section{Conclusions}

The use of institutional childbirth services remains suboptimal in the Philippines despite marked improvements in reducing maternal and child mortality and even in the proportion of women who delivered in health facilities. There are a number of factors that influence the choice of women to deliver at home, including those identified by our study: age, marital status, education, wealth index, parity, birth order, women's decision-making power, ANC visits, and emergency preparedness. Our findings suggest these factors yield similar results among the overall, urban, and rural populations, but with a relatively greater effect observed for the rural population in most of the study factors considered. Current strategies therefore need to be revisited to substantially reduce preventable deaths and narrow the gap between urban and rural women. Innovative interventions targeting women with specific characteristics as reported in our study are needed and should be considered as the country transitions to universal health care that aims equitable access for all Filipinos. Efforts should also be made to contextualize and co-create healthcare services and solutions that will motivate women to deliver in health facilities. Our study points to several recommendations that could encourage women to choose an institutional childbirth: (a) empower women to make informed healthcare decisions by making health information accessible, but at the same time targeting and involving their partners and close networks; (b) encourage women to pursue formal education with the necessary support needed from the government; (c) allow cultural birthing procedures as long as these do not conflict with scientific medical practice (e.g., massages); (d) establish additional health facilities with other services such as transport (e.g., ambulance) as these expenditures further burden the mother and discourage her from seeking care; (e) encourage positive experiences in the health facility. With the ongoing COVID-19 pandemic, it will be equally important to identify areas for improvement in the continuum of women's healthcare from pregnancy to childbirth. Providing women with the support they need and engaging them in a discussion on their choice of setting is critical in establishing trust in the healthcare system, especially at a time when health services are not easily accessible and risk-free to women in disadvantaged communities. Further studies could explore these themes in-depth using a mixedmethods approach or qualitative study design to complement our findings.

\section{Abbreviations}

aOR - Adjusted Odds Ratio

Department of Health - Department of Health

GIDA - Geographically Isolated and Disadvantaged Areas

MMR - Maternal Mortality Ratio

MNCHN - Maternal, Neonatal and Child Health and Nutrition Strategy

NDHS - National Demographic and Health Survey

NHIP - National Health Insurance Program

PSA - Philippine Statistics Authority

SDG - Sustainable Development Goals

UHC - Universal Health Care

UNDP - United Nations Development Program

WHO - World Health Organization

\section{Declarations}

\section{Ethics approval}

This study received ethics approval from the Ateneo de Manila University Research Ethics Committee (Protocol ID: SMPH DATA 2020).

\section{Availability of data and materials}

The 2017 Philippine National Demographic and Health datasets are available from the Demographic and Health Surveys Program Website (https://www.dhsprogram.com/data/available-datasets.cfm).

\section{Competing interests}

Page $14 / 17$ 
The authors declare that they have no competing interests.

\section{Funding}

This study is supported by the Ateneo de Manila University Research Council Standard Grant (Grant No. 2020-01).

\section{Authors' contributions}

AMLA, VCFP, and LST contributed to study concept and design. AMLA analyzed the data with inputs from VCFP. AMLA wrote the draft of the manuscript. All authors contributed to data interpretation, critically revised the report, and approved the final version for submission.

\section{Acknowledgements}

We thank Jeanne Christine C. Peralta, Edward Joshua M. Nuguid, and the rest of the URC-DAP Working Group for their support and insights.

\section{References}

1. Alkema L, Chou D, Hogan D, Zhang S, Moller A-B, Gemmill A, et al. Global, regional, and national levels and trends in maternal mortality between 1990 and 2015, with scenario-based projections to 2030: a systematic analysis by the UN Maternal Mortality Estimation InterAgency Group. Lancet. 2016;387:462-74.

2. Wang $\mathrm{H}$, Liddell CA, Coates MM, Mooney MD, Levitz CE, Schumacher AE, et al. Global, regional, and national levels of neonatal, infant, and under-5 mortality during 1990-2013: a systematic analysis for the Global Burden of Disease Study 2013. Lancet. 2014;384:957-79.

3. United Nations Assembly. United Nations Millennium Declaration. 2000. http://undocs.org/A/RES/55/2. Accessed 25 March 2021.

4. Countdown to 2015, Health Metrics Network. Monitoring maternal, newborn and child health: understanding key progress indicators. Geneva: World Health Organization.; 2011. https://apps.who.int/iris/handle/10665/44770. Accessed 25 March 2021.

5. Cagayan MSF, Ricarte JA, Ang-Bon RM, Llamas-Clark E. Insights on maternal health in the Philippines from national health surveys and maternal health policies. Philippine Journal of Health Research and Development. 2018;22:4.

6. United Nations Assembly. Transforming our world: the 2030 Agenda for Sustainable Development. 2015. http://www.un.org/ga/search/view_doc.asp?symbol=A/RES/70/1\&Lang=E. Accessed 25 March 2021.

7. World Health Organization. WHO recommendations: intrapartum care for a positive childbirth experience. Geneva: World Health Organization; 2018. https://www.who.int/reproductivehealth/publications/intrapartum-care-guidelines/en/. Accessed 25 March 2021.

8. Campbell OMR, Graham WJ. Strategies for reducing maternal mortality: getting on with what works. Lancet. 2006;368:1284-1299.

9. Department of Health. The MNCHN manual of operations. 2011. https://doh.gov.ph/sites/default/files/publications/MNCHNMOPMay4withECJ.pdf. Accessed 25 Apr 2021. Accessed 25 March 2021.

10. Philippine Statistics Authority. Sustainable Development Goal. 2020. https://psa.gov.ph/sdg/Philippines/baselinedata/3\%20Good\%20Health\%20and\%20Well-being. Accessed 1 May 2021.

11. Department of Health. Implementing rules and regulations of the Universal Health Care Act (Republic Act No. 11223). 2019. https://www.philhealth.gov.ph/about_us/UHC-IRR_Signed.pdf. Accessed 25 April 2021.

12. Dayrit M, Lagrada L, Picazo O, Pons M, Villaverde M. The Philippines health system review. Health Syst Transit. $2018 ; 8: 2$.

13. The World Bank. Maternal mortality ratio (modeled estimate, per 100,000 live births) - Philippines. 2017. https://data.worldbank.org/indicator/SH.STA.MMRT?locations=PH. Accessed 1 May 2021.

14. Philippine Statistics Authority, ICF International. Philippine National Demographic and Health Survey 2013. 2014. https://dhsprogram.com/pubs/pdf/FR294/FR294.pdf. Accessed 1 May 2021.

15. The World Bank. Project appraisal document on a proposed loan in the amount of US\$ 16.0 million to the Republic of the Philippines for a second Women's Health \& Safe Motherhood Project (Report 31456-PH). 2005. http://www-wds.worldbank.org/external/default/main? pagePK=64193027 \&piPK=64187937\&theSitePK=523679\&menuPK=6418751 0\&searchMenuPK = 64187282\&theSitePK = 523679\&entityID = 000090341_20 050405090650\&searchMenuPK = 64187282\&theSitePK = 523679. Accessed 28 May 2021.

16. RTI International. Luzon Health - Integrated maternal, neonatal, child health and nutrition/family planning in the Philippines. 2016. https://www.rti.org/impact/ luzonhealth-maternal-neonatal-child-health-nutrition-family-planning. Accessed 28 May 2021.

17. Gabrysch S, Campbell OM. Still too far to walk: Literature review of the determinants of delivery service use. BMC Pregnancy Childbirth. 2009;9:34.

18. Lee RB, Abellera M, Triunfante CC, Mirandilla MEL. Utilization of maternal health care services among low-income Filipino women, with special reference to Bicol Region. Asia Pac E J Health Soc Sci. 2012;1:1. 
19. Hodge A, Firth S, Bermejo R, Zeck W, Jimenez-Soto E. Utilisation of health services and the poor: deconstructing wealth-based differences in facility-based delivery in the Philippines. BMC Public Health. 2016;16:523.

20. Yamashita T, Reyes Tuliao MT, Concel Meana M, Suplido S, Llave C, Tanaka Y, et al. Utilization of healthcare services in postpartum women in the Philippines who delivered at home and the effects on their health: a cross-sectional analytical study. Int $\mathrm{J}$ Womens Health. 2017;695:700.

21. Shimazaki A, Honda S, Dulnuan MM, Chunanon JB, Matsuyama A. Factors associated with facility-based delivery in Mayoyao, Ifugao Province, Philippines. Asia Pac Fam Med. 2013;12:5.

22. Kraft AD, Nguyen K-H, Jimenez-Soto E, Hodge A. Stagnant neonatal mortality and persistent health inequality in middle-income countries: a case study of the Philippines. PLoS One. 2013;8:e53696.

23. Gouda HN, Hodge A, Bermejo R, Zeck W, Jimenez-Soto E. The impact of healthcare insurance on the utilisation of facility-based delivery for childbirth in the Philippines. PLoS One. 2016;11:e0167268.

24. Cagayan MSFS, Pepito VC, Bon RMA, Llamas-Clark EF. Experiences and satisfaction of high-risk mothers who gave birth in select facilities in Legazpi City, Philippines. International Journal of Public Health Science. 2020;9:137-144.

25. Rocca-Ihenacho L, Alonso C. Where do women birth during a pandemic? Changing perspectives on safe motherhood during the COVID-19 pandemic. J Glob Health Sci. 2020;2:e4.

26. Philippine Statistics Authority, ICF International. Philippine National Demographic and Health Survey 2017. 2018. https://psa.gov.ph/sites/default/files/PHILIPPINE\%20NATIONAL\%20DEMOGRAPHIC\%20AND\%20HEALTH\%20SURVEY\%202017_new.pdf. Accessed 22 Feb 2021.

27. Ngowi AF, Kamazima SR, Kibusi S, Gesase A, Bali T. Women's determinant factors for preferred place of delivery in Dodoma region Tanzania: a cross sectional study. Reprod Health. 2017;14:112.

28. Hailu D, Berhe H. Determinants of institutional childbirth service utilisation among women of childbearing age in urban and rural areas of Tsegedie district, Ethiopia. Midwifery. 2014;30:9.

29. Fekadu M, Regassa N. Skilled delivery care service utilization in Ethiopia: analysis of rural-urban differentials based on National Demographic and Health Survey (DHS) data. Afr H Sci. 2015;14:974.

30. Swain P, Singh P, Priyadarshini S. Determinants of home deliveries - findings from India DLHS 4 analysis. J Family Med Prim Care. 2020;9:4723.

31. Devkota B, Maskey J, Pandey AR, Karki D, Godwin P, Gartoulla P, et al. Determinants of home delivery in Nepal - A disaggregated analysis of marginalised and non-marginalised women from the 2016 Nepal Demographic and Health Survey. PLoS One. 2020;15:17.

32. Edmonds JK, Paul M, Sibley L. Determinants of place of birth decisions in uncomplicated childbirth in Bangladesh: An empirical study. Midwifery. 2012;28:554-60.

33. Abubakar S, Adamu D, Hamza R, B. Galadima J. Determinants of home delivery among women attending antenatal care in Bagwai Town, Kano Nigeria. Afr J Reprod Health. 2017;21:73-9.

34. Kusuma YS, Kaushal S, Garg R, Babu BV. Birth preparedness and determinants of birth place among migrants living in slums and slumlike pockets in Delhi, India. Sex Reprod Healthc. 2018;16:160-6.

35. Moshi FV, Mbotwa $\mathrm{CH}$. Determinants for choice of home birth over health facility birth among women of reproductive age in Tanzania: an analysis of data from the 2015-16 Tanzania Demographic and Health Survey and malaria indicator survey. BMC Pregnancy Childbirth. 2020;20:561.

36. Sobel HL, Oliveros YE, Nyunt-U S. Secondary analysis of a national health survey on factors influencing women in the Philippines to deliver at home and unattended by a healthcare professional. Int J Gynaecol Obstet. 2010;111:157-60.

37. StataCorp. Stata 14.0 IC. College Station, Tx: StataCorp; 2015.

38. Shehu CE, Ibrahim MTO, Oche MO, Nwobodo El. Determinants of place of delivery: A comparison between an urban and a rural community in Nigeria. J Public Health Epidemiol. 2016;8:91-101.

39. Ansariadi A, Manderson L. Antenatal care and women's birthing decisions in an Indonesian setting: does location matter? Rural Remote Health. 15:2959.

40. Srivastava A, Avan BI, Rajbangshi P, Bhattacharyya S. Determinants of women's satisfaction with maternal health care: a review of literature from developing countries. BMC Pregnancy Childbirth. 2015;15:97.

41. Mistry R, Galal O, Lu M. Women's autonomy and pregnancy care in rural India: A contextual analysis. Soc Sci Med. 2009;69:926-33.

42. Woldemicael G, Tenkorang EY. Women's autonomy and maternal health-seeking behavior in Ethiopia. Matern Child Health J. 2010;14:988-98. 
43. Sychareun V, Hansana V, Somphet V, Xayavong S, Phengsavanh A, Popenoe R. Reasons rural Laotians choose home deliveries over delivery at health facilities: a qualitative study. BMC Pregnancy Childbirth. 2012;12:86.

44. Fotso J-C, Ezeh AC, Essendi H. Maternal health in resource-poor urban settings: how does women's autonomy influence the utilization of obstetric care services? Reprod Health. 2009;6:9.

45. Chukwuma A, Mbachu C, McConnell M, Bossert TJ, Cohen J. The impact of monetary incentives on referrals by traditional birth attendants for postnatal care in Nigeria. BMC Pregnancy Childbirth. 2019;19:150.

46. Al-Shaikh GK, Ibrahim GH, Fayed AA, Al-Mandeel H. Grand multiparity and the possible risk of adverse maternal and neonatal outcomes: a dilemma to be deciphered. BMC Pregnancy Childbirth. 2017;17:310.

47. Delgado-Rodriguez M, Llorca J. Bias. J Epidemiol Community Health. 2004;58:635-41. 